\title{
Deslocamentos excessivos em coberturas de madeira como condicionantes de patologias
}

\author{
Excessive displacement in timber roof structures as \\ pathology conditioners
}

\author{
Arthur Filipe Freire Gomes \\ Edson Fernando Castanheira Rodrigues \\ Larissa Soriani Zanini Ribeiro Soares Silva \\ Francisco Antonio Rocco Lahr \\ André Luis Christoforo
}

\section{Resumo}

${ }^{1}$ Arthur Filipe Freire Gomes ${ }^{1}$ Universidade Federal de São Carlos Piumhi - MG - Brasil

${ }^{2}$ Edson Fernando Castanheira Rodrigues

2Universidade Federal de São Carlos São Carlos - SP - Brasil

${ }^{3}$ Larissa Soriani Zanii Ribeiro Soares Silva ${ }^{3}$ Universidade Federal de São Carlos Uberaba - MG - Brasil

${ }^{4}$ Francisco Antonio Rocco Lahr ${ }^{4}$ Universidade de São Paulo São Carlos - SP - Brasil

${ }^{5}$ André Luis Christoforo ${ }^{5}$ Universidade Federal de São Carlos São Carlos - SP - Brasil

Recebido em 05/02/20 Aceito em 22/08/20

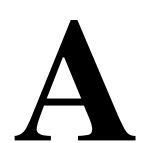

NBR 7190 (ABNT, 1997) assume o valor médio do módulo de elasticidade à compressão na direção paralela às fibras $\left(\mathrm{E}_{\mathrm{c} 0, \mathrm{~m}}\right)$ como o representativo de todas as barras de estruturas. Devido à variabilidade da madeira, tal metodologia pode resultar em valores de deslocamento superiores ao limite definido pela norma, o que pode gerar patologias na estrutura. Buscando avaliar a influência da adoção do $\mathrm{E}_{\mathrm{c} 0, \mathrm{~m}}$ no cálculo das flechas, foram realizados ensaios experimentais para obter as propriedades mecânicas da madeira branquilho. Com o auxílio de simulações numéricas e de valores experimentais, foram analisadas três tipologias (fink, bowstring e banzos paralelos) e dois vãos $(10 \mathrm{~m}$ e $20 \mathrm{~m})$. Adotando o $\mathrm{E}_{\mathrm{c} 0 \mathrm{~m}}$, foram determinadas as cargas máximas para $\mathrm{o}$ deslocamento de L/200. Posteriormente, utilizando o mesmo carregamento, foram atribuídos valores aleatórios de rigidez para cada elemento da treliça, medindo o deslocamento máximo de cada modelo. Em modelos para os quais o módulo de elasticidade foi atribuído aleatoriamente, foram observadas flechas superiores em aproximadamente $28 \%$ e $32 \%$ para vãos de $10 \mathrm{~m}$ e de $20 \mathrm{~m}$ respectivamente. Isso pode justificar algumas patologias que podem ser evitadas com a pré-classificação não destrutiva e a colocação racional das peças em regiões mais solicitadas.

Palavras-chave: MEF. Compressão paralela às fibras. Rigidez. Treliça. Análise não linear.

\section{Abstract}

The NBR 7190 (ABNT, 1997) assumes the mean value of the elasticity module to compression in the direction parallel to grain $\left(E_{c 0, m}\right)$ as being the representative for all roof structure bars. Due to the variability of woods, such methodology can result in displacement values higher than the limit defined by the standard, which can generate pathologies in the structure. In order to evaluate the influence of the use of $E_{c 0, m}$ on the displacement calculation, experimental tests were carried out to obtain the mechanical properties of the wood species Sebastiania commersoniana (Baillon) Smith \& Downs. With the aid of numerical simulations and experimental values, three typologies (fink, bowstring and parallel chord) and two spans $(10 \mathrm{~m}$ and $20 \mathrm{~m})$ were analysed. Adopting $E_{c 0, m}$, the maximum loads for a displacement of $L / 200$ were determined. After, random stiffness values were assigned to each element of the truss, measuring the maximum displacement of each model. In models where the modulus of elasticity was randomly assigned, superior arrows were observed in approximately $28 \%$ and $32 \%$ for spans of $10 \mathrm{~m}$ and $20 \mathrm{~m}$, respectively. It may explain some pathologies that can be avoided with nondestructive pre-classification and the rational placement of parts in the most vulnerable areas.

Keywords: FEM. Compression parallel to grain. Stiffness. Truss. Nonlinear analysis.

GOMES, A. F. F.; RODRIGUES, E. F. C.; SILVA, L. S. Z. R. S.; LAHR, F. A. R.; CHRISTOFORO, A. L. Deslocamentos excessivos em coberturas de madeira como condicionantes de patologias. Ambiente Construído, Porto Alegre, v. 21, n. 3, p. 147-158, jul./set. 2021.

ISSN 1678-8621 Associação Nacional de Tecnologia do Ambiente Construído. http://dx.doi.org/10.1590/s1678-86212021000300543 


\section{Introdução}

A madeira é um material de grande potencial de uso na construção civil e pode ser procedente de florestas naturais ou plantadas (MAZURANA et al., 2011; MATOS et al., 2012; TER STEEGE et al., 2016; TUISIMA-CORAL et al., 2017). Entre os materiais comumente utilizados, a madeira é o único renovável, expressa elevada relação entre a resistência e o peso, e sua produção gera poucos impactos ambientais.

Para a execução de estruturas de madeira com segurança e economia, é necessário o conhecimento das propriedades mecânicas das espécies (TREVISAN et al., 2007; LIN; FU, 2008). Dessa forma, é fundamental a realização de ensaios com a finalidade de caracterizar as propriedades utilizadas no dimensionamento estrutural. A etapa de realização de ensaios é relativamente rápida quando comparada à fase de confecção de corpos de provas. Ademais, há a necessidade da utilização de equipamentos e instrumentos capazes de aferir as propriedades mecânicas, os quais têm custo elevado para a aquisição e a manutenção.

Entre as propriedades mecânicas avaliadas, segundo a NBR 7190 (ABNT, 1997), o módulo de elasticidade $\left(\mathrm{E}_{\mathrm{c} 0}\right)$ e a resistência à compressão na direção paralela às fibras consistem nos valores fundamentais para a elaboração de projetos estruturais (YANG; EVANS, 2003; SCANAVACA JÚNIOR; GARCIA, 2004). Na verificação dos estados limites de serviço, a norma brasileira indica o valor médio $\left(\mathrm{E}_{\mathrm{c} 0 \mathrm{~m}}\right)$ do módulo de elasticidade à compressão na direção paralela às fibras.

De acordo com Yang e Evans (2003), quando se trata de madeiras procedentes de plantações comerciais de rápido crescimento, que têm como objetivo suprir a demanda comercial, o conhecimento das propriedades mecânicas, sobretudo do módulo de elasticidade, é de suma importância, visto que, frequentemente, esse material contém grande proporção de madeira juvenil e pode não apresentar propriedades mecânicas satisfatórias para determinados usos (HEIN et al., 2009), fato que pode culminar em diversas patologias, como deslocamentos e deformações excessivas em estruturas treliçadas de cobertura.

As peças de madeira são largamente empregadas como elementos estruturais em coberturas. Os procedimentos de projeto e cálculo estão muito bem definidos nas normativas, entretanto é comum a observação de estruturas de madeira destinadas a coberturas (tesouras ou treliças) com anomalias que prejudicam seu uso e sua segurança, como discutido nas pesquisas de Andrade Júnior et al. (2012, 2014) e Paludo et al. (2017).

Existem diversos sistemas estruturais de coberturas, sendo os principais arcos, vigas e treliças (CARNIELLE, 2011). Segundo Kripka et al. (2013), uma treliça é constituída por elementos lineares (barras) que podem estar dispostos em diversos planos, estruturas bidimensionais ou tridimensionais. Usualmente, a treliça é construída em madeira ou aço, o que a torna relativamente leve, sendo indicada para superar grandes vãos ou superar carregamentos elevados.

Entre os diversos tipos de geometrias de treliças, podem-se destacar o tipo fink (Figura 1a) (RIBEIRO et al., 2016), o tipo bowstring (Figura 1b) e banzos paralelos (Figura 1c) (CALIL JÚNIOR; DIAS, 1997). A treliça fink é uma variação da treliça belga, com montantes e diagonais centrais de menor comprimento. É geralmente utilizada para vãos entre $20 \mathrm{~m}$ e $30 \mathrm{~m}$, com relação $\mathrm{h} / \mathrm{L}$ variando entre $1 / 5 \mathrm{e} 1 / 4$. A treliça do tipo bowstring possui a parte superior semelhante a arcos, e o banzo inferior reto, sendo utilizada para vãos de até $30 \mathrm{~m}$, com relação $\mathrm{h} / \mathrm{L}$ variando entre $1 / 6$ e 1/10. A treliça do tipo banzos paralelos é adotada quando existe a necessidade do formato retangular do telhado, sendo utilizada para vãos de até $20 \mathrm{~m}$, com relação h/L variando entre $1 / 6$ e $1 / 10$.

Nas estruturas treliçadas de madeira para coberturas, uma decorrência das patologias consiste nos deslocamentos perceptivelmente exagerados (ANDRADE JÚNIOR et al., 2014), que podem ser provenientes da perda nas propriedades de rigidez e resistência dos elementos estruturais. Para a avaliação dessa manifestação patológica, é necessário avaliar o carregamento último de colapso e sua resposta, força aplicada versus deslocamento. Dependendo das características estruturais, essa resposta pode ser linear ou não linear.

A análise estrutural linear clássica pressupõe proporcionalidade entre carga e deslocamento. As condições para que essa proporcionalidade se verifique são a resposta elástica linear do material e pequenos deslocamentos. A condição de resposta linear do material pode ser garantida limitando-se os níveis de tensão e deformação. Todavia, no comportamento não linear não há proporcionalidade entre o carregamento aplicado e as deformações e/ou deslocamentos, sendo necessário o emprego de técnicas que possam considerar de maneira apropriada os efeitos da não linearidade geométrica (SOUZA, 2015).

148 Gomes, A. F. F.; Rodrigues, E. F. C.; Silva, L. S. Z. R. S.; Lahr, F. A. R.; Christoforo, A. L. 


\section{Figura 1 - Estruturas treliçadas}

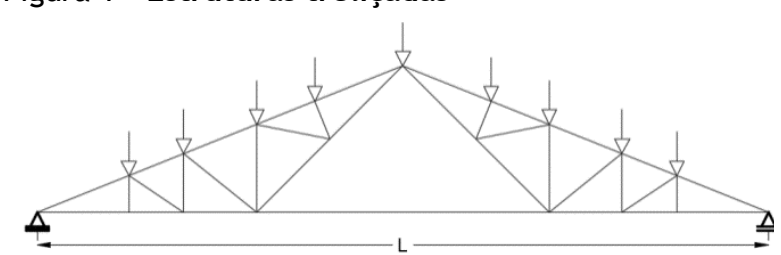

(a) Fink

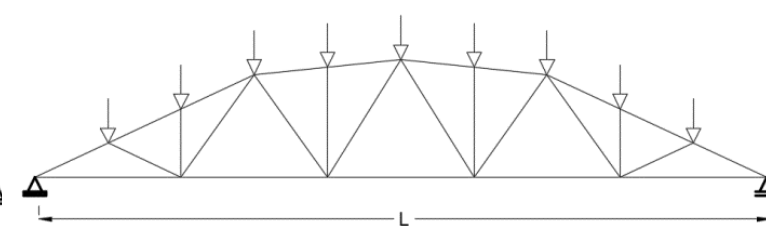

(b) Bowstring

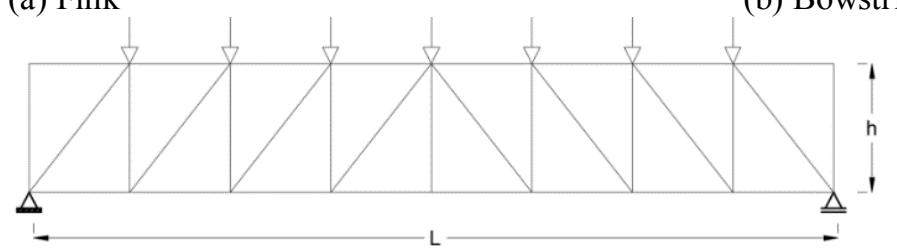

(c) Banzos paralelos

Entretanto, na fase de projeto, uma possível fonte de patologia pode advir da adoção do módulo de elasticidade médio. A adoção de um mesmo módulo de elasticidade para todas as barras componentes da estrutura, como preconiza a norma brasileira NBR 7190 (ABNT, 1997), pode resultar em deslocamentos máximos superiores aos de referência estabelecidos para a condição de estado limite de serviço. Na condição real, as barras têm módulos de elasticidade distintos, e a falta de uma pré-classificação mecânica não destrutiva, o que é raro nos projetos estruturais, impede que os elementos mais solicitados do projeto recebam as barras com os maiores valores dos módulos e elasticidade.

Além disso, há ausência de informações a respeito das manifestações patológicas oriundas das condições estabelecidas no dimensionamento de estruturas de cobertura de madeira pela norma brasileira NBR 7190 (ABNT, 1997), assim como também ocorre em outros documentos normativos. Portanto, esta pesquisa objetivou avaliar os deslocamentos máximos de treliças considerando-se ora o valor médio do módulo de elasticidade médio à compressão na direção paralela às fibras para todas as barras, ora os valores reais dos módulos de elasticidade aleatoriamente alocados nas barras. Com isso, foi possível comparar os valores de deslocamentos obtidos utilizando a metodologia preconizada pela normativa (adoção do $\mathrm{E}_{\mathrm{c} 0, \mathrm{~m}}$ para todas as barras) com uma situação mais próxima da realidade, em que ocorre a variação das propriedades e em que a alocação das peças é frequentemente realizada de modo aleatório. Com o auxílio de um software para análises numéricas (linear física e não linear geométrica), foram avaliados os deslocamentos de três tipos de geometria treliçadas (fink, bowstring e banzos paralelos) e vãos de $10 \mathrm{~m}$ e $20 \mathrm{~m}$.

\section{Materiais e métodos}

Para a determinação da resistência (f) e da rigidez (E), foram realizados ensaios de compressão e tração na direção paralela às fibras em 32 corpos de prova de espécie de branquilho (Sebastiania commersoniana). Os ensaios foram executados segundo a metodologia apresentada no projeto de norma PNBR 02:126.10-001-1 (ABNT, 2013), que consiste na atualização da norma brasileira NBR 7190 (ABNT, 1997).

As madeiras apresentaram teor de umidade de $12 \%$, sendo este o teor de equilíbrio da madeira e, portanto, definido pela norma NBR 7190 como a condição padrão de umidade para o cálculo de propriedades da madeira. Os ensaios experimentais foram conduzidos no Laboratório de Madeiras e de Estruturas de Madeira, do Departamento de Engenharia de Estruturas, da Escola de Engenharia de São Carlos (LaMEM/SET/EESC), da Universidade de São Paulo (USP).

O programa experimental consistiu em 16 corpos de prova com seção transversal quadrada com $5 \mathrm{~cm}$ de lado e comprimento igual a $15 \mathrm{~cm}$ (Figura 2a), na máquina universal Amsler (Figura 2b), seguindo o ciclo de carga e descarga apresentado na PNBR 02:126.10-001-1 (ABNT, 2013). Nesse ciclo, o corpo de prova é carregado até $50 \%$ de sua força de ruptura, permanecendo por $30 \mathrm{~s}$ nesse nível de carregamento. Em seguida, o carregamento é reduzido a até $10 \%$ da força de ruptura, permanecendo nesse nível de carregamento por mais $30 \mathrm{~s}$. Após essa fase, a força é aumentada até a ruptura do corpo de prova. Foram utilizados medidores mecânicos de deformação com sensibilidade de $0,001 \mathrm{~mm}$ para as leituras em todos os ensaios. 
Figura 2 - Corpos de prova posicionados nas máquinas de ensaio para os testes de compressão (a) e de tração (b) na direção paralela às fibras da madeira de branquilho

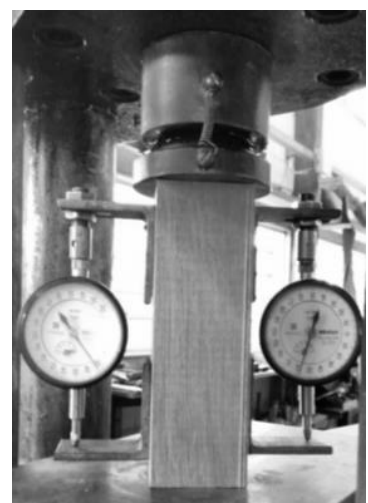

(a)

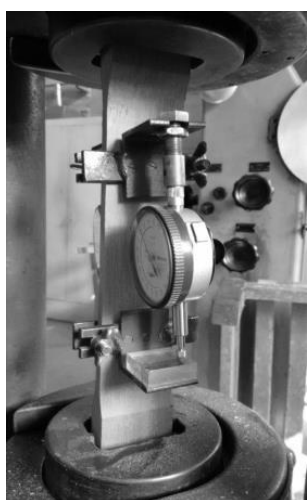

(b)

Obtidas as propriedades mecânicas experimentalmente, realizou-se a análise numérica dos deslocamentos das estruturas de madeira. Para o referente estudo, foram avaliadas três tipologias de treliças (fink, bowstring e banzos paralelos) com comprimento de vãos de $10 \mathrm{~m}$ e $20 \mathrm{~m}$. Para a treliça de $10 \mathrm{~m}$ foi adotada a relação $\mathrm{h} / \mathrm{L}$ de $1 / 5$ para todos os tipos de treliças utilizados neste trabalho. Para vãos de $20 \mathrm{~m}$ adotou-se a relação $\mathrm{h} / \mathrm{L}$ para a tipologia fink de 1/5; e para as tipologias bowstring e banzos paralelos foi de $1 / 6,25$, valores dentro dos limites recomendados para cada tipologia.

Além do vão, foram realizadas onze variações dos valores de módulo de elasticidade em cada barra da treliça. Uma análise dos deslocamentos foi realizada adotando-se o valor médio do módulo de elasticidade à compressão na direção paralela às fibras, visto que esse é o valor indicado pela PNBR 02-126.10-001-1 (ABNT, 2013). Para os outros dez modelos, foram adotados valores de módulo de elasticidade provenientes dos ensaios realizados, dispostos aleatoriamente nas estruturas.

Para a definição das cargas a serem utilizadas, não foram feitas combinações de ações ou consideração do efeito do vento. Os carregamentos foram definidos de forma que o valor da flecha fosse próximo ao estado limite de serviço $(\mathrm{L} / 200)$ no modelo de referência $\left(\mathrm{E}_{\mathrm{c} 0, \mathrm{~m}}\right)$.

Para vãos de $10 \mathrm{~m}$, foi considerado o espaçamento entre treliças de $9 \mathrm{~m}$ com telhas cerâmicas, que teve seu peso por área adotado juntamente com a estrutura de madeira igual a $0,7 \mathrm{KN} / \mathrm{m}$ aplicado na treliça mais solicitada, com área de influência igual a $10 \mathrm{~m}$ x $9 \mathrm{~m}$. A força resistida para o pior caso de cada tipo de treliça foi de $63 \mathrm{kN}$, que foi dividida para 9 nós para os tipos fink e bowstring, e para 7 nós para o tipo banzos paralelos.

Para vãos de $20 \mathrm{~m}$, considerou-se o espaçamento entre as treliças de $12,5 \mathrm{~m}$, estando presente nessa área uma cobertura com telhas cerâmicas. Foi adotada uma carga de trabalho de $0,7 \mathrm{kN} / \mathrm{m}$ aplicada em toda a área de influência $(12,5 \mathrm{~m}$ x $20 \mathrm{~m}$ ) de uma treliça central (maiores valores solicitantes). A força resistida por cada treliça foi de $175 \mathrm{kN}$, sendo dividida igualmente em 9 nós para os tipos fink (Figura 1a) e bowstring (Figura 1b), e em 7 nós para o tipo banzos paralelos (Figura 1c).

Para a definição da seção transversal para cada modelo, a esbeltez $(\lambda)$ das peças comprimidas foi limitada a 140, valor máximo permitido pela norma brasileira NBR 7190 (ABNT, 1997). Após a modelagem das estruturas, foram obtidas todas as medidas das peças da treliça e, então, foi definida a seção transversal para a barra com maior valor de esbeltez. Visando analisar estritamente a influência da tipologia, do vão e do módulo de elasticidade, a mesma seção transversal foi adotada para todas as barras da estrutura, sendo escolhidas seções quadradas com lados múltiplos de $10 \mathrm{~mm}$. As seções adotadas são mostradas na Tabela 1.

Para simular o comportamento estrutural de treliças de madeira, foram desenvolvidos modelos de elementos finitos no software SAP2000. Realizaram-se análises não lineares geométricas para verificar os deslocamentos máximos da estrutura. As treliças foram consideradas isostáticas, sendo o apoio da esquerda responsável por restringir a translação na vertical e na horizontal, e o apoio da direita impedindo o movimento na vertical. Foram utilizados elementos de barra para representar a estrutura, sendo realizada uma análise não linear geométrica. Sendo a treliça isostática, o colapso de uma única barra provoca a ruína de toda a estrutura. Para cada vão e tipologia das estruturas treliçadas, foi escolhida a barra com maior esbeltez submetida à compressão para realizar uma análise de flambagem. Com o auxílio do software

150 Gomes, A. F. F.; Rodrigues, E. F. C.; Silva, L. S. Z. R. S.; Lahr, F. A. R.; Christoforo, A. L. 
Abaqus, foram gerados seis modelos numéricos (um para cada barra), sendo realizadas duas análises: flambagem elástica e flambagem não linear plástica.

O comportamento da madeira foi considerado linear até a carga de ruptura, seguido de um patamar plástico. Tal comportamento foi baseado no resultado do trabalho de Felipe et al. (2019). A carga de flambagem elástica foi realizada por meio da análise Buckle, disponível no software Abaqus. Como resposta, tem-se a carga crítica de flambagem elástica (autovalor) e a configuração deformada do pilar (autovetor). Com esses dados, é realizada a segunda análise, em que o autovetor é utilizado como dado de entrada. Nessa etapa, são introduzidas as imperfeições geométricas inserindo a palavra-chave imperfection no arquivo de entrada. Utilizando a deformada obtida na primeira análise, os deslocamentos dos pontos discretizados são multiplicados por um fator representativo da imperfeição. Assim, o modelo computacional utilizado para a segunda análise apresenta uma curvatura inicial com a mesma configuração do primeiro autovetor, sendo o deslocamento máximo igual ao valor da imperfeição introduzida. No presente estudo, foi utilizado um valor de $10 \%$ da menor dimensão da seção transversal.

Foi realizado um estudo de sensibilidade de malha com o tamanho máximo de $30 \mathrm{~mm}, 20 \mathrm{~mm}, 10 \mathrm{~mm}$ e 5 mm para a barra de maior comprimento submetida à compressão de cada treliça. Foram comparadas as cargas de flambagem teórica (carga de Euler) com a carga obtida através do modelo numérico. A barra foi considerada biapoiada, tendo a aplicação de uma carga pontual em uma das extremidades. A Figura 3 mostra os valores obtidos para a barra da treliça bowstring de $20 \mathrm{~m}$ para os diferentes tamanhos de malha. Após alguns testes com outros elementos, o utilizado foi o S4R (elemento de casca com quatro nós e integração reduzida), pois apresentou alta precisão e curto tempo de processamento. A Figura 4 apresenta o detalhamento da malha utilizada, e a Figura 5 a posição indeformada, o primeiro (considerado para implementar as imperfeições iniciais) e o terceiro modo de flambagem. Destaca-se que o segundo modo de flambagem é idêntico ao primeiro por se tratar de seções quadradas.

Tabela 1 - Seções adotadas

\begin{tabular}{c|c|c|c}
\hline Vão & fink & bowstring & banzos paralelos \\
\hline $10 \mathrm{~m}$ & $5 \times 5 \mathrm{~cm}$ & $5 \times 5 \mathrm{~cm}$ & $4 \times 4 \mathrm{~cm}$ \\
$20 \mathrm{~m}$ & $9 \times 9 \mathrm{~cm}$ & $8 \times 8 \mathrm{~cm}$ & $8 \times 8 \mathrm{~cm}$ \\
\hline
\end{tabular}

Figura 3 - Estudo de sensibilidade da malha

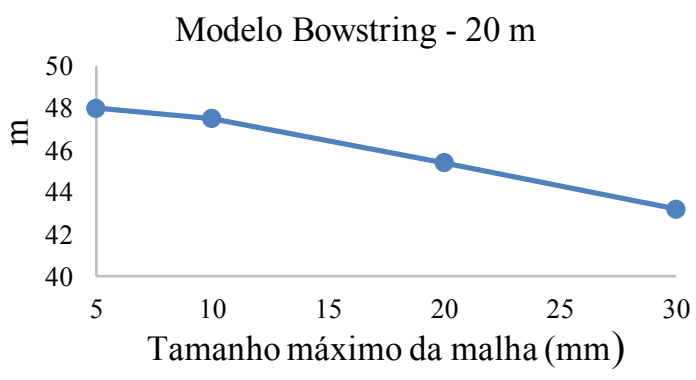

Figura 4 - Malha utilizada

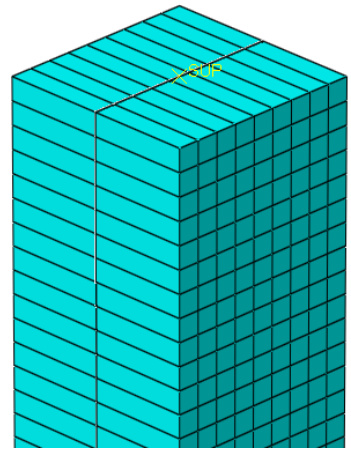


Figura 5 - Modos de flambagem

(a) Pilar na posição indeformada Nota: Legenda:

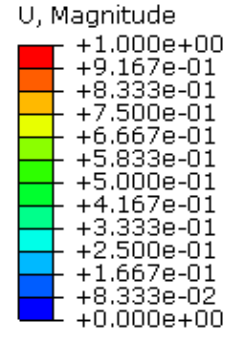

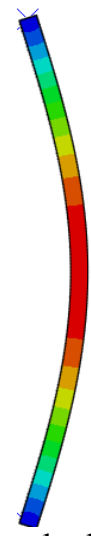

(b) Primeiro modo de flambagem

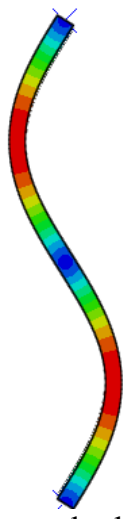

(c) Terceiro modo de flambagem

Ao total, foram feitos 66 modelos computacionais no software SAP2000 para analisar as flechas máximas, e 6 modelos (12 análises) no software Abaqus para avaliar a flambagem elástica e a flambagem não linear nas barras comprimidas de maior esbeltez. Com as análises realizadas no Abaqus, foi possível avaliar a possibilidade do colapso da treliça isostática, tendo em vista que a barra analisada poderia se tornar instável com uma carga inferior à solicitada pela carga aplicada na estrutura.

A partir dos valores das flechas obtidos pelas simulações, foi elaborado o intervalo de confiança da média (ao nível de 95\% de confiabilidade), buscando estabelecer a margem de incerteza dos valores de deslocamentos obtidos. Com essa metodologia, foi possível verificar se as flechas obtidas, considerando-se o módulo de elasticidade médio $\left(\mathrm{E}_{\mathrm{c} 0, \mathrm{~m}}\right)$, pertencem ou não aos intervalos encontrados. Assim, foi possível avaliar se as flechas nos modelos com módulos de elasticidade arbitrariamente alocados seriam superiores a L/200, sugerindo que a utilização do módulo de elasticidade médio à compressão na direção paralela às fibras como fator de projeto é contra a segurança, podendo gerar flechas excessivas e, consequentemente, tornando a estrutura suscetível a maiores patologias. Além disso, através da capacidade de carga das barras obtida pelas simulações, foi ponderada a importância da análise de elementos isolados da treliça, principalmente em estruturas isostáticas.

\section{Resultados e discussão}

Na Tabela 2 é apresentada a síntese dos resultados obtidos através dos ensaios experimentais, sendo apresentados os valores médios, os coeficientes de variação $(\mathrm{CV})$, os valores máximos e mínimos, e os intervalos de confiança (IC).

Segundo o projeto de atualização da norma brasileira PNBR 02:126.10-001-1 (ABNT, 2013), pode ser considerada a equivalência entre os módulos de elasticidade à tração $\left(\mathrm{E}_{\mathrm{t}, 0}\right)$ e à compressão $\left(\mathrm{E}_{\mathrm{c}, 0}\right)$ na direção paralela. Com o objetivo de verificar a correspondência entre tais valores, foi realizada uma análise de variância (ANOVA) com o auxílio do software Minitab versão 18, ao nível de 5\% de significância. Os resultados obtidos são apresentados na Tabela 3.

Da Tabela 3, o P-valor da ANOVA ser superior ao nível de significância adotado $(5 \%$ - 0,05$)$ implica que os valores médios do $\mathrm{E}_{\mathrm{c}, 0}$ e do $\mathrm{E}_{\mathrm{t}, 0}$ são considerados estatisticamente equivalentes, assim como prediz a norma brasileira NBR 7190 (ABNT, 1997). Como informação adicional, os valores característicos da resistência à compressão $\left(\mathrm{f}_{\mathrm{c} 0, \mathrm{k}}\right)$ e da resistência à tração $\left(\mathrm{f}_{\mathrm{t} 0, \mathrm{k}}\right)$ na direção paralela às fibras da madeira de branquilho calculados segundo a norma brasileira NBR 7190 foram iguais a 45,64 MPa e a 67,49 MPa respectivamente,

152 Gomes, A. F. F.; Rodrigues, E. F. C.; Silva, L. S. Z. R. S.; Lahr, F. A. R.; Christoforo, A. L. 
implicando que a relação $\mathrm{fc} 0, \mathrm{k} / \mathrm{ft} 0 . \mathrm{k}=0,77$ não foi atendida para essa espécie no conjunto de resultados obtidos $\left(\mathrm{f}_{\mathrm{c} 0, \mathrm{k}} / \mathrm{f}_{\mathrm{t} 0, \mathrm{k}}=0,68\right)$.

Utilizando os valores das propriedades obtidas pelos ensaios, foram realizadas as simulações numéricas das treliças. A Figura 6 ilustra as deformadas das estruturas fink, bowstring e banzos paralelos, com vão de $20 \mathrm{~m}$ e considerando-se o módulo de elasticidade médio à compressão na direção paralela às fibras. Na Tabela 4 são apresentados os valores das flechas $(\mathrm{cm})$ para as estruturas treliçadas com vãos $(\mathrm{L})$ de $10 \mathrm{~m}$ e $20 \mathrm{~m}$. Como supracitado, os valores de módulo de elasticidade foram atribuídos aleatoriamente, como acontece normalmente nas obras de cobertura.

Os resultados obtidos para os três tipos de treliças com vãos de $10 \mathrm{~m}$ (Tabela 4) revelam que, para quase todas as combinações de barras e módulos de elasticidade, foram encontrados valores de flechas superiores ao valor de flecha (L/200) obtido considerando-se o valor médio do módulo de elasticidade. As treliças bowstring e banzos paralelos foram as que apresentaram maiores flechas, 7,33 cm para ambas, ou seja, valor $31,79 \%$ superior ao resultado que considerou o módulo de elasticidade médio. Dos valores máximos, a treliça com menor flecha foi a do tipo fink, $7,32 \mathrm{~cm}$, sendo esse valor significativo por ser $31,69 \%$ superior ao valor obtido quando se utiliza o $\mathrm{E}_{\mathrm{c} 0, \mathrm{~m}}$.

Tabela 2 - Síntese dos resultados dos valores das propriedades de resistência e de rigidez da madeira de branquilho

\begin{tabular}{c|c|c|c|c}
\hline Estat. & $\mathbf{f}_{\mathbf{c} 0}(\mathbf{M P a})$ & $\mathbf{f}_{\mathbf{t} 0}(\mathbf{M P a})$ & $\mathbf{E}_{\mathbf{c} 0}(\mathbf{M P a})$ & $\mathbf{E}_{\mathbf{t} 0}(\mathbf{M P a})$ \\
\hline Média & 48,16 & 95,47 & 14.667 & 14.416 \\
CV $(\%)$ & 8,69 & 18,97 & 12,8 & 16,5 \\
Mín. & 40,87 & 53,44 & 9.917 & 10.269 \\
Máx. & 59,18 & 131,00 & 17.904 & 19.162 \\
IC $(95 \%)$ & $(46,66 ; 49,68)$ & $(88,64 ; 102,00)$ & $(13.990 ; 15.346)$ & $(13.560 ; 15.272)$ \\
\hline
\end{tabular}

Tabela 3 - Resultados da ANOVA para a verificação da equivalência entre os valores de rigidez à compressão $(E c 0)$ e à tração (Et0) na direção paralela às fibras da madeira de branquilho

\begin{tabular}{c|c|c|c|c|c}
\hline Fonte & GL & SQ (Aj.) & QM (Aj.) & Valor-F & P-Valor \\
\hline Ensaio & 1 & 1011173 & 1011173 & 0,22 & 0,64 \\
Erro & 62 & 284288423 & 4585297 & & \\
Total & 63 & 285299596 & & & \\
\hline
\end{tabular}

Nota: Legenda:

$\mathrm{GL}$ - graus de liberdade;

SQ - soma dos quadrados; e

QM - quadrado médio.

Figura 6 - Estruturas treliçadas fink (a), bowstring (b) e banzos paralelos (c) simuladas com vão de $20 \mathrm{~m}$ e com módulo de elasticidade médio na compressão paralela às fibras

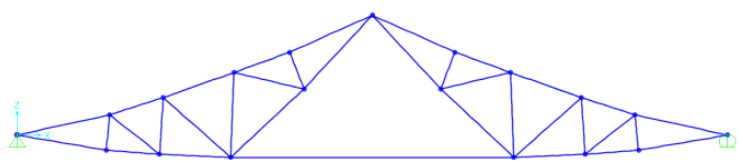

(a)

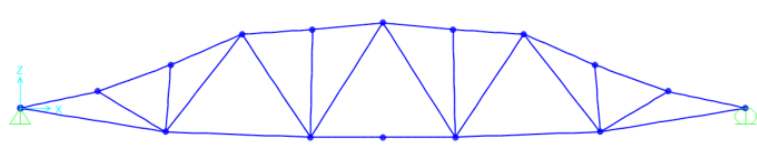

(b)

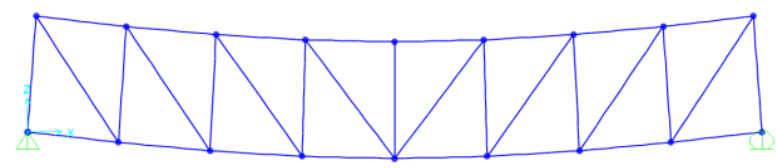

(c)

Fonte: SAP2000 (2005). 
Tabela 4 - Flechas $(\mathrm{cm})$ para as treliças com vãos de $\mathrm{L}=10 \mathrm{~m}$ e $\mathrm{L}=20 \mathrm{~m}$

\begin{tabular}{c|c|c|c|c|c|c|c}
\hline \multicolumn{3}{c}{ Flechas (cm) para L= 10 m } & \multicolumn{3}{c}{ Flechas (cm) para L = 20 m } \\
\hline Simulações & fink & bowstring & $\mathbf{B P}$ & Simulações & fink & bowstring & $\mathbf{B P}$ \\
\hline $\mathbf{0}$ * (NBR 7190) & $\mathbf{5}$ & $\mathbf{5}$ & $\mathbf{5}$ & $\mathbf{0} *$ (NBR 7190) & $\mathbf{9 , 9}$ & $\mathbf{9 , 2}$ & $\mathbf{6 , 9 0}$ \\
\hline 1 & 5,22 & 5,23 & 5,46 & 1 & 10,60 & 9,60 & 7,60 \\
\hline 2 & 5,91 & 4,94 & 5,5 & 2 & 11,80 & 9,00 & 7,50 \\
\hline 3 & 5,56 & 6,08 & 4,85 & 3 & 11,20 & 11,10 & 6,60 \\
\hline 4 & 5,89 & 6,32 & 6,94 & 4 & 11,70 & 11,50 & 9,20 \\
\hline 5 & 6,48 & 5,04 & 5,37 & 5 & 12,90 & 9,20 & 7,10 \\
\hline 6 & 5,24 & 6,15 & 6,00 & 6 & 10,50 & 11,20 & 7,90 \\
\hline 7 & 5,19 & 7,02 & 5,76 & 7 & 10,20 & 12,80 & 7,70 \\
\hline 8 & 4,87 & 5,64 & 4,71 & 8 & 9,70 & 10,30 & 6,40 \\
\hline 9 & 4,52 & 6,51 & 5,44 & 9 & 9,00 & 11,90 & 7,30 \\
\hline 10 & 7,32 & 7,33 & 7,33 & 10 & 9,60 & 8,6 & 7,40 \\
\hline Média - Ealeat & 5,62 & 6,03 & 5,74 & Média - Ealeat & 10,72 & 10,52 & 7,47 \\
\hline Mínimo - Ealeat & 4,52 & 4,94 & 4,71 & Mínimo - Ealeat & 9,00 & 8,60 & 6,40 \\
\hline Máximo - Ealeat & 7,32 & 7,33 & 7,33 & Máximo - Ealeat & 12,90 & 12,80 & 9,20 \\
\hline
\end{tabular}

Nota: BP refere-se a banzos paralelos e *aos valores da flexa utilizando o $\mathrm{E}_{\mathrm{c} 0, \mathrm{~m}}$.

Verificou-se que as flechas para os três tipos de treliças de vãos de $20 \mathrm{~m}$ também foram em quase sua totalidade superiores aos valores de flechas obtidos considerando-se o módulo de elasticidade médio e o limite (L/200) recomendado na norma NBR 7190 (ABNT, 1997). O maior valor de flecha foi proveniente da simulação de número 7 (treliça do tipo bowstring), $12,80 \mathrm{~cm}$, ou seja, $28,13 \%$ superior quando comparado à flecha com o módulo de elasticidade médio. Dos valores máximos, a treliça que apresentou menor flecha foi a do tipo de banzos paralelos, com $9,20 \mathrm{~cm}$, todavia esse valor representa $25,00 \%$ de acréscimo quando comparado ao valor de deslocamento obtido considerando-se o módulo de elasticidade médio na compressão paralela e, proporcionalmente, é a maior discrepância encontrada entre as treliças de $20 \mathrm{~m}$ de vão. A treliça de banzos paralelos foi calculada com área das seções transversais de seus montantes, banzos superiores e inferiores maiores do que nos casos das outras duas treliças, visto que, se suas peças recebessem a mesma seção transversal que nos outros dois casos, seria gerado um índice de esbeltez maior que 140 nas barras mais esbeltas compridas, o que, segundo a NBR 7190, deve ser evitado.

A Figura 7 apresenta os gráficos dos intervalos de confiança da média (95\% de confiabilidade) referente às flechas $(\mathrm{d}$, em $\mathrm{cm})$ dos três tipos de treliças com vãos de $10 \mathrm{~m}$ e $20 \mathrm{~m}$ avaliadas nesta pesquisa, simuladas considerando-se os módulos de elasticidade na compressão paralela dispostos aleatoriamente nas barras dessas estruturas.

Dos gráficos apresentados da Figura 7, observou-se que os valores das flechas obtidos com o módulo de elasticidade médio $E_{\mathrm{c} 0, \mathrm{~m}}$ não estão compreendidos entre os limites inferiores e superiores dos intervalos de confiança na maioria dos casos, com exceção apenas para a treliça do tipo fink de $20 \mathrm{~m}$ de vão. Esse fato constata como os módulos de elasticidade reais interferem de forma preponderante nas flechas, sendo possíveis causas de patologias.

Foram escolhidas seis barras, sendo uma de cada tipologia e vão, para calcular a capacidade de carga de tais elementos. Utilizando-se a malha de tamanho máximo de $10 \mathrm{~mm}$, o elemento do tipo $\mathrm{S} 4 \mathrm{R}$ e o modelo constitutivo supracitado, a Tabela 5 apresenta os valores obtidos para as barras. Como pode ser observado, o modelo numérico apresenta ótima concordância com os valores teóricos.

Nas Figuras 8 e 9 são destacados os valores de cálculo nas barras mais esbeltas (esbeltez acima de 80) dos três tipos de treliças e para os dois vãos utilizados para cada uma. Os gráficos mostram uma fase linear seguida de um comportamento não linear da estrutura. O comportamento linear é apresentado até carregamentos próximos à capacidade de carga da peça. Com valores próximos à capacidade de carga, os elementos são regidos por comportamentos não lineares. Com carregamentos próximos à carga máxima, as barras são submetidas a maiores valores de deslocamentos (principalmente laterais), e com isso os efeitos de segunda ordem exercem maior influência no comportamento do elemento estrutural.

Com base nas análises feitas nos softwares SAP2000 e Abaqus, foi possível estimar as cargas de cada barra na treliça, assim como a capacidade de carga da barra mais esbelta de cada tipologia. A capacidade resistente da barra foi comparada ao valor em que o elemento está submetido na estrutura treliçada. A comparação das

154 Gomes, A. F. F.; Rodrigues, E. F. C.; Silva, L. S. Z. R. S.; Lahr, F. A. R.; Christoforo, A. L. 
cargas máximas da barra mais esbelta determinadas por uma análise linear e uma análise não linear geométrica é apresentada na Tabela 6. Observa-se a relevância da consideração de imperfeições geométricas (analise não linear) devido à considerável perda de capacidade resistente, com diminuição de aproximadamente $22 \%$ da carga máxima. Das seis barras analisadas, quatro foram submetidas a esforços superiores a sua capacidade de carga. Desse modo, a barra está sujeita à instabilidade, assim como toda a treliça, por se tratar de uma estrutura isostática.

Figura 7 - Intervalos de confiança das médias das flechas para as treliças com vãos de (a) 10 m e (b) 20 m

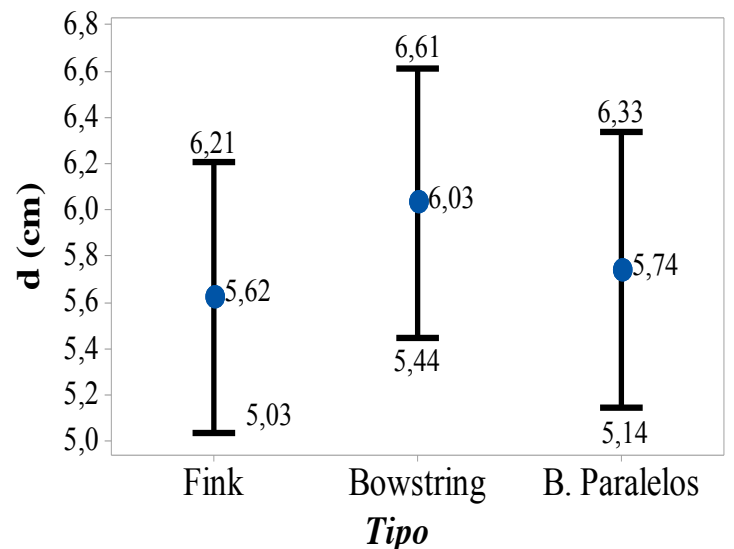

(a)

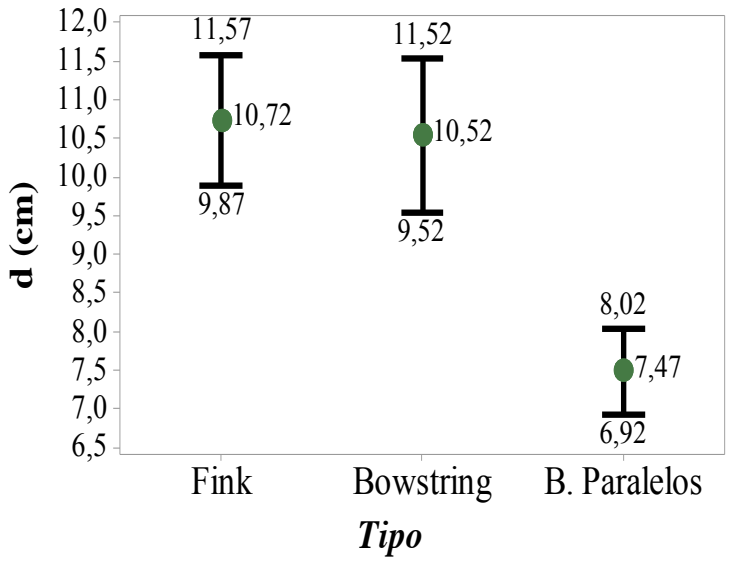

(b)

Fonte: Minitab (2019).

Tabela 5 - Cálculo de flambagem elástica

\begin{tabular}{c|c|c|c}
\hline Tipos & $\begin{array}{c}\text { Carga de flambagem } \\
\text { elástica teórica }(\mathbf{k N})\end{array}$ & $\begin{array}{c}\text { Carga de flambagem } \\
\text { elástica numérica (kN) }\end{array}$ & $\begin{array}{c}\text { Diferença } \\
\text { percentual }\end{array}$ \\
\hline fink 20 m & 109,4 & 107,9 & $-1,4 \%$ \\
bowstring 20 m & 54,9 & 54,0 & $-1,6 \%$ \\
BP 20 m & 48,3 & 47,5 & $-1,6 \%$ \\
fink 10 m & 41,7 & 40,0 & $-4,0 \%$ \\
bowstring 10 m & 33,5 & 32,2 & $-3,9 \%$ \\
BP 10 m & 12,1 & 11,9 & $-1,5 \%$ \\
\hline
\end{tabular}

Nota: BP refere-se a banzos paralelos.

Figura 8 - Gráfico força $x$ deslocamento para treliças de $10 \mathrm{~m}$

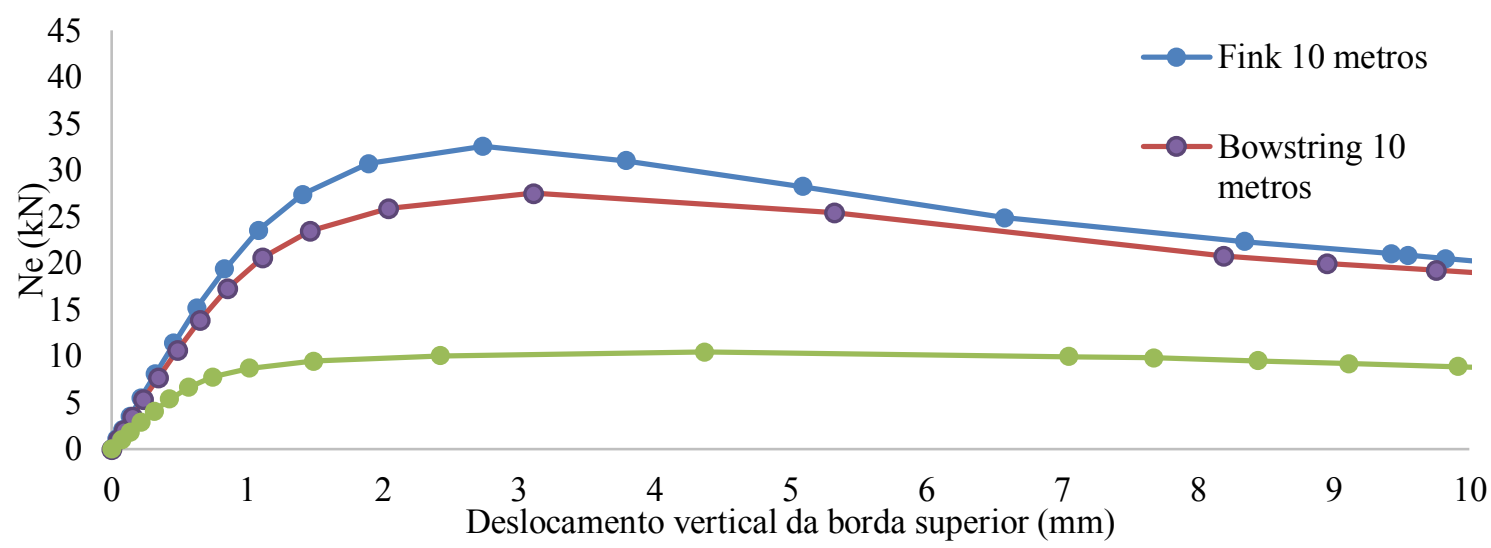


Figura 9 - Gráfico força $\mathrm{x}$ deslocamento para treliças de $20 \mathrm{~m}$

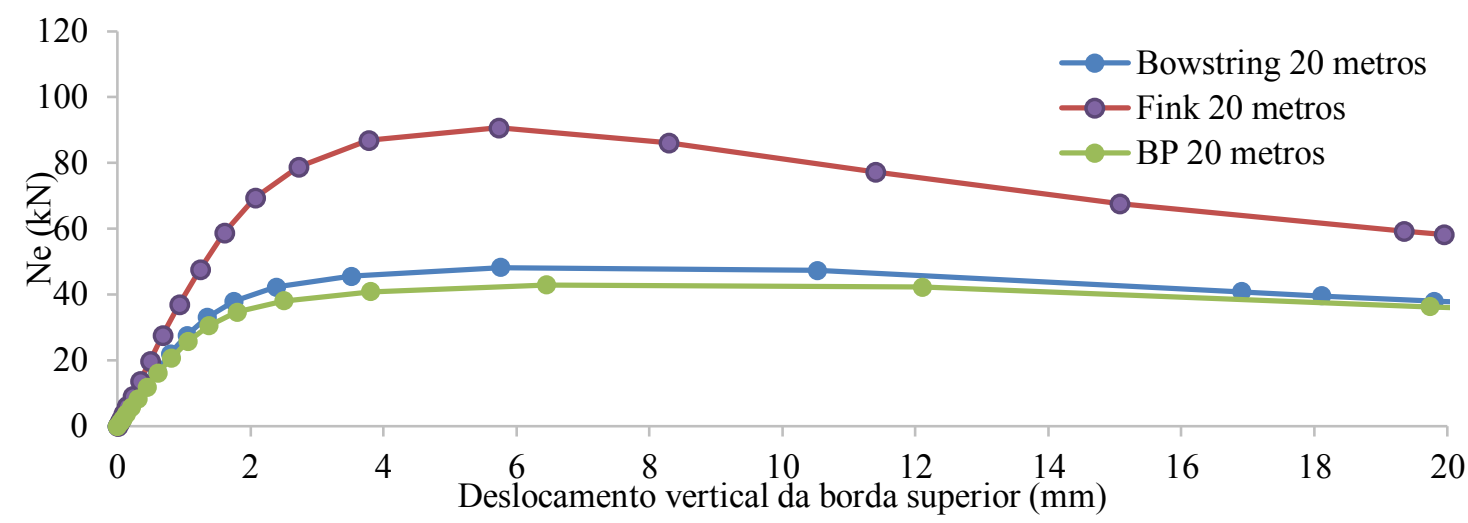

Tabela 6 - Capacidade de carga

\begin{tabular}{c|c|c|c}
\hline Tipos & $\begin{array}{c}\text { Carga de flambagem } \\
\text { elástica numérica }(\mathbf{k N})\end{array}$ & $\begin{array}{c}\text { Carga de flambagem } \\
\text { não linear }(\mathbf{k N})\end{array}$ & Diferença \\
\hline fink 20 m & 107,9 & 90,7 & $-17,04 \%$ \\
bowstring 20 m & 54,0 & 48,2 & $-12,26 \%$ \\
BP 20 m & 47,5 & 42,9 & $-11,09 \%$ \\
fink 10 m & 40,0 & 32,5 & $-21,92 \%$ \\
bowstring 10 m & 32,2 & 27,5 & $-17,90 \%$ \\
BP 10 m & 11,9 & 10,4 & $-13,43 \%$ \\
\hline
\end{tabular}

Nota: BP refere-se a banzos paralelos.

\section{Conclusões}

Devido à grande variabilidade das propriedades mecânicas da madeira, a adoção de um valor médio se mostra uma maneira simplista na determinação das flechas em estruturas de madeira. Com base nos resultados e nas análises realizadas neste estudo, pode-se observar que o cálculo dos deslocamentos da estrutura utilizando valor médio do módulo de elasticidade à compressão na direção paralela às fibras, conforme preconiza a NBR 7190 (ABNT, 1997), pode prever deslocamentos inferiores aos que a estrutura pode apresentar em situações reais.

Na maioria dos casos em que os módulos de elasticidade foram atribuídos de maneira aleatória, os valores de flecha máxima foram superiores aos obtidos utilizando-se o valor médio. Para o vão de $10 \mathrm{~m}$, o valor chegou a ser $31,79 \%$ superior, enquanto para vãos de $20 \mathrm{~m}$ os valores chegaram a ser $28,13 \%$ superiores. Tais diferenças em deslocamentos podem justificar as diversas manifestações patológicas em estruturas de cobertura e em estruturas adjacentes.

Nas análises de cada barra, foram constatadas que quatro das seis barras apresentaram valores de capacidade de carga inferiores aos que estariam submetidos nas treliças, resultando em seu colapso e, consequentemente, no colapso da estrutura. Além disso, foram obtidos valores até $21,92 \%$ inferiores ao valor obtido pela fórmula de Euler, o que demostra a necessidade uma análise individual dos elementos da treliça.

Uma prática para evitar problemas com flechas excessivas consiste na adoção de técnicas não destrutivas para a obtenção do módulo de elasticidade da madeira, como o ultrassom (MINÁ et al., 2004). Dessa maneira, é possível posicionar peças mais rígidas em posições mais solicitadas, evitando flechas excessivas e talvez superiores ao limite especificado por norma e evitando patologias devido ao deslocamento excessivo da estrutura.

\section{Referências}

ANDRADE JÚNIOR, J. R. et al. Avaliação das condições estruturais de telhados construídos com madeira brasileira nativa. Revista Madeira: Arquitetura e Engenharia, v. 13, p. 1-10, 2012.

156 Gomes, A. F. F.; Rodrigues, E. F. C.; Silva, L. S. Z. R. S.; Lahr, F. A. R.; Christoforo, A. L. 
ANDRADE JÚNIOR, J. R. et al. Avaliação das estruturas de cobertura em madeira de um galpão de estoque de produtos químicos. Ambiente Construído, Porto Alegre, v. 14, n. 3, p. 75-85, jul./set. 2014.

ASSOCIAÇÃO BRASILEIRA DE NORMAS TÉCNICAS. NBR 7190: projeto de estruturas de madeira. Rio de Janeiro, 1997.

ASSOCIAÇÃO BRASILEIRA DE NORMAS TÉCNICAS. PNBR - 02:126.10-001-1: madeiras: método de ensaio de caracterização de corpos de prova isentos de defeitos para madeiras tropicais. Rio de Janeiro, 2013.

CALIL JÚNIOR, C.; DIAS, A. A. Utilização da madeira em construções rurais. Revista Brasileira de Engenharia Agrícola e Ambiental, v. 1, n. 1, p. 71-77, 1997.

CARNIELLE, R. O. A. Caracterização das construções com madeira em Uberlândia: patologias, projetos e detalhes. Uberlândia, 2011. Dissertação (Mestrado em Engenharia Civil) - Universidade Federal de Uberlândia, Uberlândia, 2011.

FELIPE, T. R. C. et al. Análise não linear física e geométrica em ensaio de compressão paralela: caso de estudo na madeira marupá. Revista Interscientia, v. 7, n. 2, p. 41-49, 2019.

HEIN, P. R. G. et al. Estimativa da resistência e da elasticidade à compressão paralela às fibras da madeira de Eucalyptus grandis e E. urophylla usando a espectroscopia no infravermelho próximo. Scientia Forestalis, v. 37, n. 82, p. 119-129, 2009.

KRIPKA, M. et al. Minimização do peso de treliças de alumínio pela otimização simultânea da seção transversal dos elementos e da geometria: análise numérica e validação experimental. Revista Eletrônica de Engenharia Civil, v. 7, n. 2, p. 19-26, 2013.

LIN, L.; FU, F. Segregation of Eucalyptus lumbers for finger-jointed using longitudinal vibration. Chinese Forestry Science and Technology, v. 7, n. 1, p. 64-70, 2008.

MATOS, G. S. B. et al. Desenvolvimento Inicial e estado nutricional de clones de eucalipto no nordeste do Pará. Acta Amazônica, v. 42, n. 2, p. 491-500, 2012.

MAZURANA, M. et al. Balanço de nutrientes em povoamentos de Eucalyptus saligna implantado sobre cambissolo háplico no RS. Revista Brasileira de Engenharia Agrícola e Ambiental, v. 15, n. 9, p. 924 930, 2011.

MINÁ, A. J. S. et al. Avaliação não destrutiva de postes de madeira por meio de ultrassom. Scientia Forestalis, v. 65, p. 188-196, 2004.

MINITAB. Software de análise de dados e estatística. Versão 18. 2019.

PALUDO, D. F. et al. Timber use in truss structures for roof (howe type - 8 to 18 meters). International Journal of Materials Engineering, v. 7, p. 93-99, 2017.

RIBEIRO, A. C. S. et al. Comparative study of wood consumption in structures of concrete roof. International Journal of Materials Engineering, v. 6, n. 3, p. 85-91, 2016.

SAP2000. Static and Dynamic Finite Element Analysis of Structural Analysis Program, Advanced 10.01. Computers and Structures, 2005.

SCANAVACA JÚNIOR, L.; GARCIA, SCANAVACA JÚNIOR, L.; GARCIA, J. N. Determinação das propriedades físicas e mecânicas da madeira de Eucalyptus urophylla. Scientia Forestalis, v. 65, p. 120-129, 2004.

SOUZA, L. A. F. Análise não linear física de treliças planas por elementos finitos considerando a mecânica do dano.In: ENCONTRO INTERNACIONAL DE PRODUÇÃO CIENTÍFICA UNICESUMAR, 9., Maringá, 2015. Anais [...] Maringá, 2015.

TER STEEGE, H. et al. The discovery of the Amazonian tree flora with an update checklist of all known tree taxa. Scientific Reports, v. 6, n. 29549, p. 1-15, 2016.

TREVISAN, T. et al. Avaliação de propriedades físicas e mecânicas da madeira de cinco espécies florestais em função da deterioração em dois ambientes. Revista Árvore, v. 31, n. 1, p. 93-101, 2007.

TUISIMA-CORAL, L. L. et al. Variation in wood physical properties within stems of Guazuma crinita, a timber tree species in the Peruvian Amazon. Maderas y Bosques, v. 23, n. 1, p. 53-61, 2017. 
YANG, J. L.; EVANS, R. Prediction of MOE of Eucalyptus wood from microfibril angle and density. Holz als Roh- und Werkstoff, v. 61, p. 449-452, 2003.

\section{Agradecimentos}

O presente trabalho foi realizado com apoio da Coordenação de Aperfeiçoamento de Pessoal de Nível Superior - Brasil (CAPES) - Código de Financiamento 001. Essa pesquisa ainda contou com o apoio do CNPq pela concessão de bolsa (processo 309058/2019-8). Além disso, os autores são gratos ao Laboratório de Madeiras e de Estruturas de Madeira, da Escola de Engenharia de São Carlos (LaMEM/SET/EESC), da Universidade de São Paulo (USP) por ter colaborado na realização dos ensaios experimentais.

\footnotetext{
Arthur Filipe Freire Gomes

Departamento de Engenharia Civil | Universidade Federal de São Carlos | Rua Floriano Peixoto, 808, Bela Vista | Piumhi - MG - Brasil | CEP 37925-000 | Tel.: (37) 99939-6892 | E-mail: arthurfreire2009@gmail.com
}

Edson Fernando Castanheira Rodrigues

Departamento de Engenharia Civil Universidade Federal de São Carlos | Rua José Gullo, 120, Quitinete 05, Vila Marina | São Carlos - SP Brasil | CEP 13566-360 | Tel.: (16) 99152-7447 | E-mail: edson.engcivil.projetos@gmail.com

Larissa Soriani Zanii Ribeiro Soares Silva

Departamento de Engenharia Civil | Universidade Federal de São Carlos | Rua João Pinheiro, 2279, Boa Vista | Uberaba - MG - Brasil | CEP 38870-000 | Tel.: (34) 99978-3714 | E-mail: larissazanini@iftm.edu.br

\section{Francisco Antonio Rocco Lahr}

Departamento de Engenharia de Estruturas, Escola de Engenharia | Universidade de São Paulo | Av. Trabalhador Sãocarlense, 400, Centro | São Carlos - SP - Brasil | CEP 13566-590 | Tel.: (16) 3373-8206 | E-mail: frocco@sc.usp.br

\section{André Luis Christoforo}

Departamento de Engenharia Civil | Universidade Federal de São Carlos | Rodovia Washington Luís, km 235, SP-310 | São Carlos - SP Brasil | CEP 13565-905 | Tel.: (16) 98259-6211 | E-mail: christoforoal@yahoo.com.br

\section{Ambiente Construído}

Revista da Associação Nacional de Tecnologia do Ambiente Construído Av. Osvaldo Aranha, $99-3^{\circ}$ andar, Centro

Porto Alegre - RS - Brasil

$$
\text { CEP } 90035-190
$$

Telefone: +55 (51) 3308-4084

www.seer.ufrgs.br/ambienteconstruido

www.scielo.br/ac

E-mail: ambienteconstruido@ufrgs.br 